\section{Doxorubicin Effect on Myocardial Metabolism as a Prerequisite for Subsequent Development of Cardiac Toxicity: Are There Unsuspected Confounders?}

TO THE EDITOR: We have enthusiastically read the meticulous and well-conducted retrospective study by Bauckneht et al. published in the October issue of The Journal of Nuclear Medicine (1). The authors assessed the role of ${ }^{18} \mathrm{~F}$-FDG PET/CT in the prediction of doxorubicin cardiac toxicity in 69 patients treated with a chemotherapy regimen for Hodgkin lymphoma (HL); furthermore, they investigated the possible dose-dependent nature of doxorubicin toxicity in 15 athymic mice and concluded that in patients undergoing doxorubicin administration, not only is myocardial ${ }^{18} \mathrm{~F}$-FDG uptake increased but also that low ${ }^{18} \mathrm{~F}-\mathrm{FDG}$ uptake before chemotherapy may predict the development of cardiac toxicity.

Myocardial cells protect themselves from hypoxic state, reducing contractile function with the downregulation of hypoxia and mitochondrial oxidative metabolism through the "glucosefatty acid cycle" (2), and ${ }^{18}$ F-FDG uptake may be a useful tool to identify a myocardial metabolic switch subsequent to cellular damage (3).

Several published retrospective and preclinical studies, some already cited by Bauckneht et al. $(4,5)$, suggest the opportunity of investigating the correlation between changes in myocardial ${ }^{18} \mathrm{~F}$ FDG uptake in pre- and postchemotherapy or radiotherapy evaluation and subsequent development of cardiac toxicity $(6,7)$.

We also observed in a preliminary study that cardiac uptake of ${ }^{18} \mathrm{~F}-\mathrm{FDG}$ could increase during chemotherapy (8). Our first hypothesis was that in patients undergoing chemotherapy some elements may modify the variable avidity for glucose (9) and shift the myocardial metabolism from $\beta$-oxidation of fatty acids to glycolysis, also considering the possible role of steroids (for iatrogenic hyperglycemia) and granulocyte colony-stimulating factor (for insulinlike effects). We tested our hypothesis retrospectively in a group of HL patients $(n=24)$ treated with a regimen of adriamycin, bleomycin, vincristine, dacarbazine (ABVD) plus $20 \mathrm{mg}$ of dexamethasone, excluding patients with antecedent cardiovascular disease, diabetes, and previous chemotherapy or mediastinal irradiation and identifying 10 patients who received from 50 to $575 \mathrm{mg}$ of steroids additional to the standard (extra-steroids group).

All patients underwent ${ }^{18} \mathrm{~F}-\mathrm{FDG} \mathrm{PET} / \mathrm{CT}$ at staging, interim, and final evaluation: no significant differences were found between different scans in patients' body weight and glycemia levels at ${ }^{18} \mathrm{~F}$-FDG injection. We observed also an incremental trend in cardiac $\mathrm{SUV}_{\max }$ at staging, interim, and final evaluation, and we found a significant association between ${ }^{18} \mathrm{~F}$-FDG uptake and extrasteroid administration $(P=0.005)$, suggesting a strong, independent (and possibly transient) correlation between administration of extra steroids and this phenomenon.

The correlation between cardiac toxicity after chemotherapy, in particular after anthracycline administration, or radiotherapy and myocardial ${ }^{18} \mathrm{~F}$-FDG uptake is a current study argument.

Given the aforementioned data, it is our opinion that ${ }^{18} \mathrm{~F}-\mathrm{FDG}$ PET/CT may be a useful biomarker of cardiac toxicity, but only after first clarifying the role of other factors that may occur as confounders in ${ }^{18}$ F-FDG uptake, such as steroid administration.

\section{REFERENCES}

1. Bauckneht M, Ferrarazzo G, Fiz F, et al. Doxorubicin effect on myocardial metabolism as a prerequisite for subsequent development of cardiac toxicity: a translational ${ }^{18}$ F-FDG PET/CT observation. J Nucl Med. 2017;58:1638-1645.

2. Randle PJ, Garland PB, Hales CN, Newsholme EA. The glucose fatty-acid cycle: its role in insulin sensitivity and the metabolic disturbances of diabetes mellitus. Lancet. 1963;1:785-789.

3. Dilsizian V, Narula J. Atlas of Nuclear Cardiology. 4th ed. New York, NY: Springer; 2013.

4. Borde C, Kand P, Basu S. Enhanced myocardial fluorodeoxyglucose uptake following adriamycin-based therapy: evidence of early chemotherapeutic cardiotoxicity? World J Radiol. 2012;4:220-223.

5. Gorla AK, Sood A, Prakash G, Parmar M, Mittal BR. Substantial increase in myocardial FDG uptake on interim PET/CT may be an early sign of adriamycininduced cardiotoxicity. Clin Nucl Med. 2016;41:462-463.

6. Evans JD, Gomez DR, Chang JY, et al. Cardiac ${ }^{18} \mathrm{~F}$-fluorodeoxyglucose uptake on positron emission tomography after thoracic stereotactic body radiation therapy. Radiother Oncol. 2013;109:82-88.

7. Basu S, Borde C, Kand P. Increasing cardiac ${ }^{18}$ F-fluorodeoxyglucose (FDG) uptake on PET-CT as a biomarker for cardiotoxicity of chemoradiotherapy in cancer: a myth or a reality? Radiother Oncol. 2014;112:451-452.

8. Parente A, Finessi M, Nicolotti D, et al. Cardiac FDG uptake increases after ABVD (adryamicine, bleomycine, vincristine, dacarbazine) and support therapy with steroids and G-CSF: an observation on 24 patients with newly-diagnosed Hodgkin's disease. Eur J Nucl Med Mol Imaging. 2012;39(suppl 2):S469.

9. Osborne MT, Hulten EA, Venkatesh LM, et al. Patient preparation for cardiac fluorine-18 fluorodeoxyglucose positron emission tomography imaging of inflammation. J Nucl Cardiol. 2017;24:86-99.

Monica Finessi*
Daniele G. Nicolotti
Gianni Bisi
Désirée Deandreis
*Division of Nuclear Medicine
Department of Medical Sciences
AOU Città della Salute e della Scienza
University of Turin, Italy
Corso Dogliotti 14, 10126 Torino
E-mail: finessimonica@ @mail.com

Published online Jan. 11, 2018.

DOI: 10.2967/jnumed.117.205856

REPLY: We are grateful to Finessi et al. for their letter, which raises a crucial topic. We agree that many possible confounding factors may challenge the interplay between anthracycline-induced cardiotoxicity and myocardial ${ }^{18} \mathrm{~F}-\mathrm{FDG}$ accumulation. Among them, steroids might profoundly interfere with myocardial metabolism, increasing insulin resistance, reducing free fatty acid serum levels, and consequently potentially affecting myocardial ${ }^{18} \mathrm{~F}$-FDG uptake (1).

On the other hand, the relevance of corticosteroids nicely fits with our hypothesis about the existence of a peculiar ${ }^{18} \mathrm{~F}-\mathrm{FDG}$ metabolic pathway located in the endoplasmic reticulum and regulated by hexose-6-phosphate dehydrogenase (H6PD) (2). This enzyme represents the unique reticular source of the reduced nicotinamide adenine dinucleotide phosphate (NADPH) moieties needed for cortisone activation $(3,4)$ in the same endoplasmic reticulum by 11 betahydroxysteroid dehydrogenase type 1 (11ß-HSD1). In this line, the increased use of NADPH reductive power caused by the administration of pharmacologic cortisone doses might "transiently" increase H6PD activity and thus myocardial ${ }^{18}$ F-FDG uptake. However, this variable should have played a minor role in our main observation (5) for the following reasons. 